\title{
Analysis and visualisation of RDF resources in Ondex
}

Catherine Canevet

catherine.canevet@bbsrc.ac.uk 


\section{Motivation}

- Biomedical resources provide info on the Semantic Web

O Potential to advance biomedical research

$\circ$ Need for tools

- Data reduction

- Data visualization

- Interactive analysis capabilities 


\section{Ondex: a platform to exploit the Semantic Web}

1) all information can be represented as a graph

2) all elements of the graph can be annotated with ontologies

3) data model conformant to Semantic Web framework, in particular to RDF

$\rightarrow$ Ondex ideally positioned 


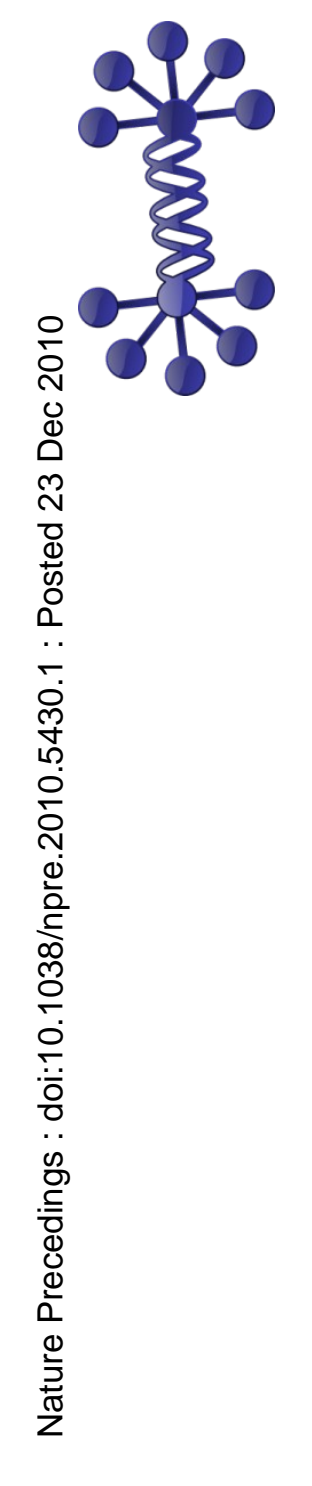

\section{Data integration and visualisation in Ondex}
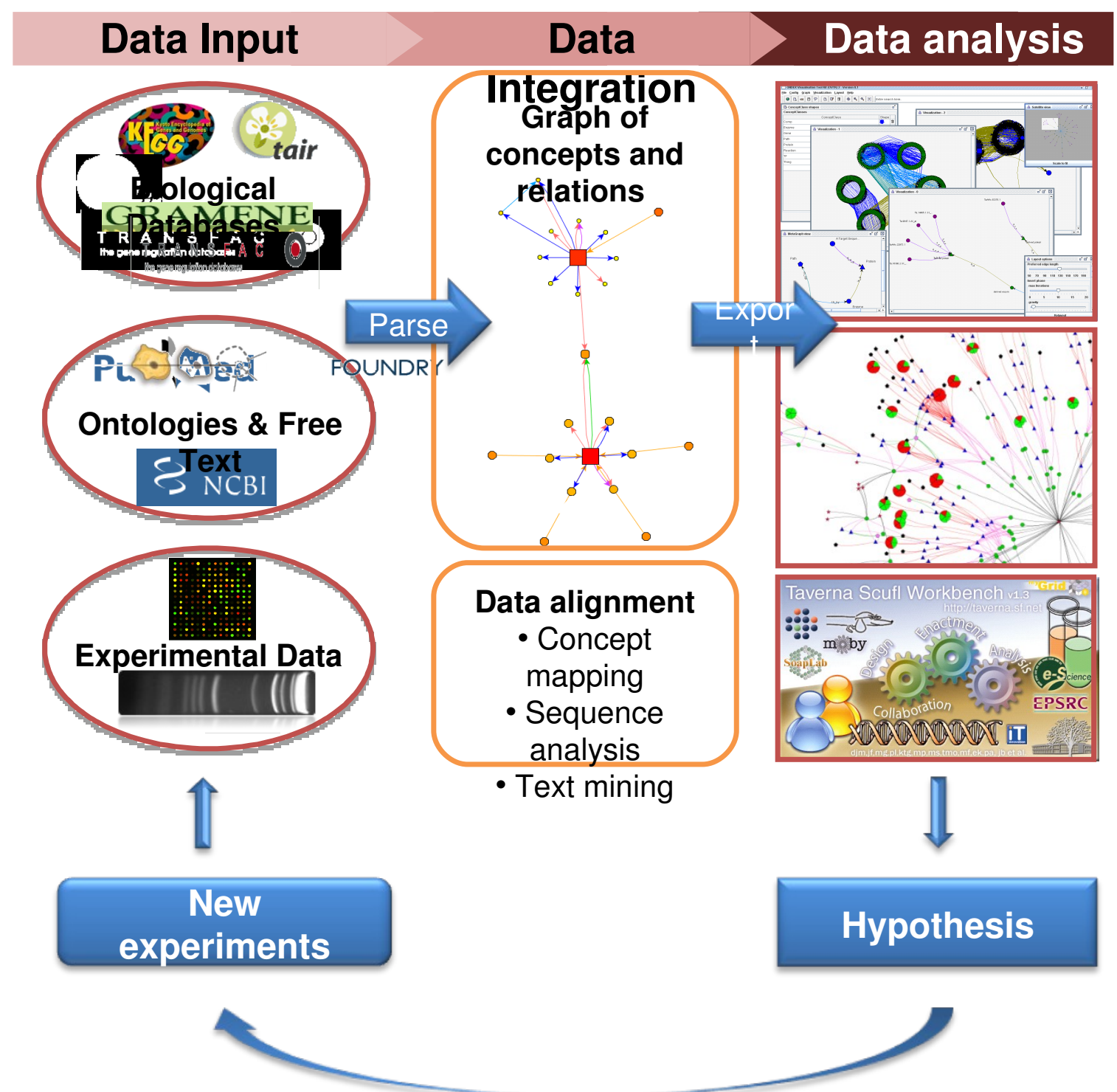


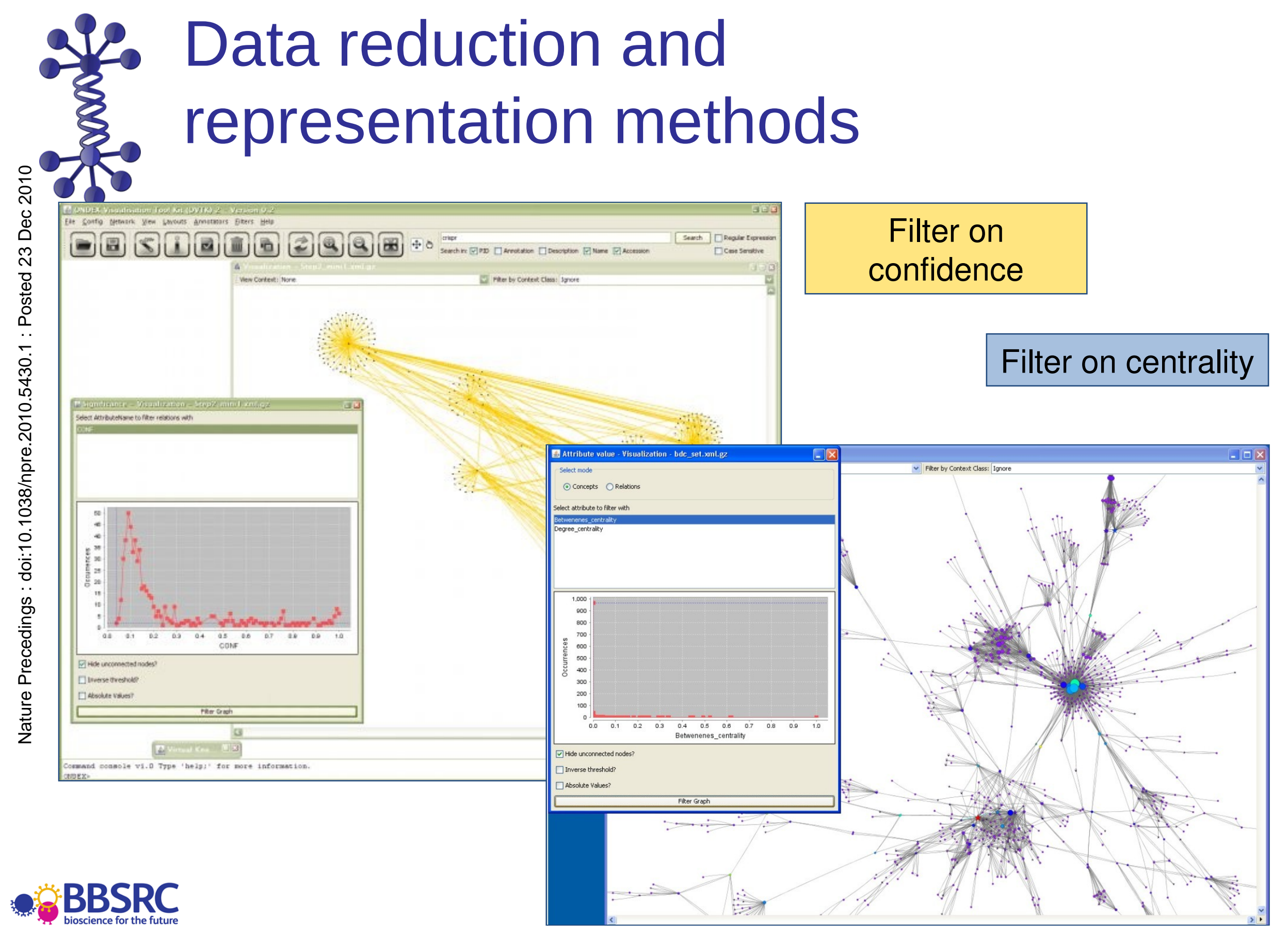


Configure Help

\section{$\square$ Export}

+ Experimental

+ Discontinued

$\checkmark$ Stable

OXL Export

Report writer

Filter

+ Discontinued

+ Stable

Q Experimental

Eaph

Papping

of Stable

. Concept accession-based mapping (Memory-efficient)

9 Inparanoid

+ Experimental

Porser

क्षि Stable

Aracyc2 (Release 6, October 14, 209)

.. BCSDB (pre-release BCSDB-3 gamma version)

-. Brenda specific SBML Parser (BRENDA release online since 2nd July 2010)

$\dot{0}$ EXPASY ENZYME (4/10/2009)

FASTA file parser

5. KEGG parser (Release 52.1, December 1, 2009)

KEGG parser, latest (Release 53.0, January 1, 2010)

@ OXL Parser

d. UniProt (UniProt release July2010)

Experimental

Geatisitics

of Experimental

Tansformer

Experimental

S stable

$\because$ Relation Collapser

응

号

六

త্ঠ

ㄴ.

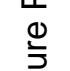

Argents | Documentation | Required data | Comments |

a subset of the TAIR9 database parsing genes, proteins, domains

Annotation

If true also $\mathrm{GO}$ annotations, protein domain

information and UniProt accessions will be parsed

InputDir

Graph id
Graph that will be operated on by this plugin.
Absolute path to input directory
New |

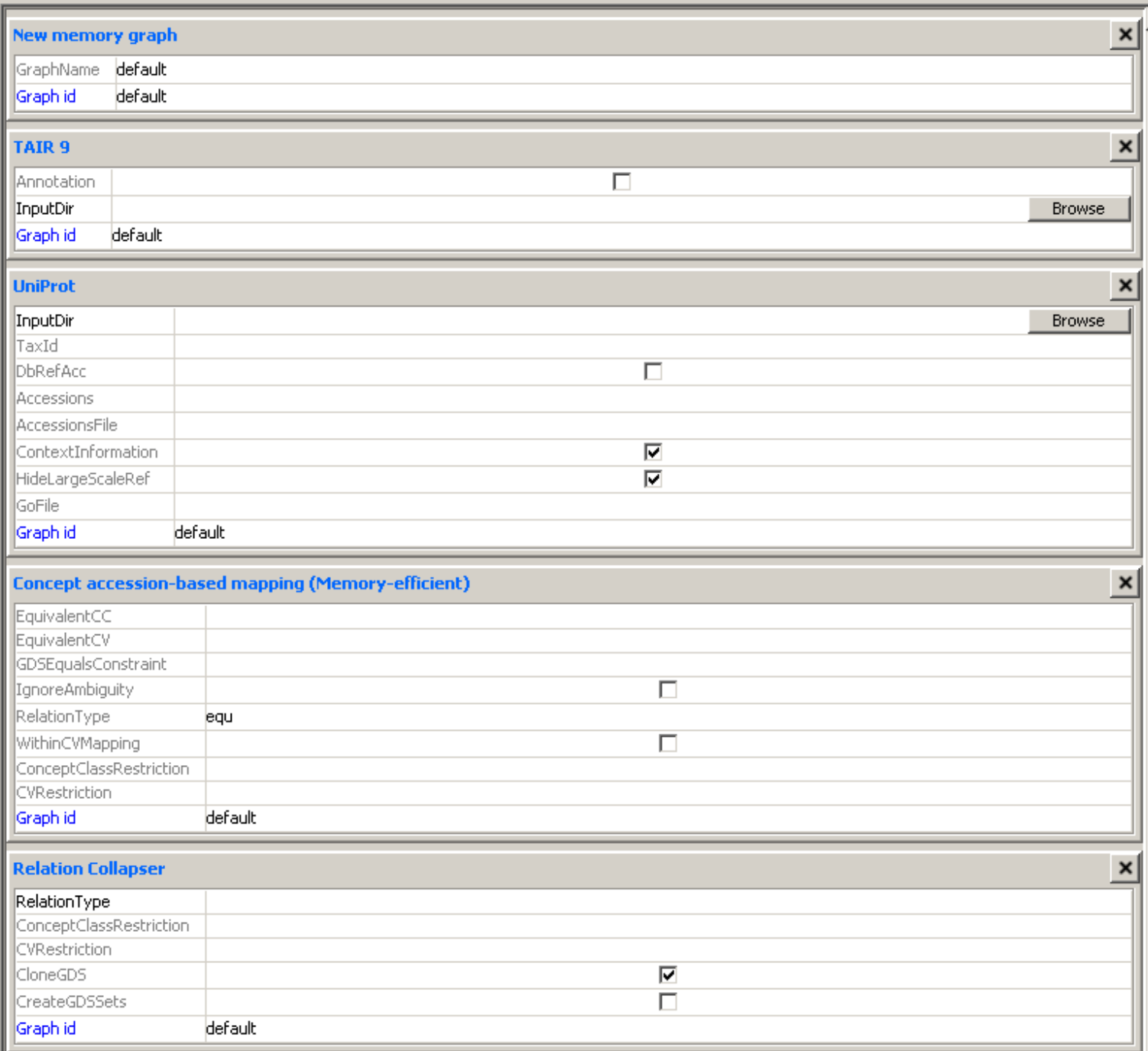

OXL Export

ExcludeConceptsofConceptClass

ExcludeRelationsofRelationType

ExcludeGDSWithAttribute

IncludeOnlyGDSAttribute

IncludeOnlyConceptClass

IncludeOnlyRelationType

Pretty

ExportIsolatedConcepts

Gzip 


\section{Demo}

- Interactive importer based on SPARQL

$\circ$ Query-driven construction of datasets

$\circ$ Bring information from different RDF data resources into Ondex

- Welcome feedback on our prototype 


\section{Data used in demo integrated by Keywan Hassani-Pak}

Input

45778 predicted poplar genes

Data Integration

PoplarCyc, Poplar TFDB

Comparative Genomics

BLAST against UniProt Plants HMMer against Pfam Inparanoid to Arabidopsis Autom. Function Prediction Poplar KB Gene, Plant and Trait Ontology Text Mining

Co-occurrence of genes and traits in Medline abstracts

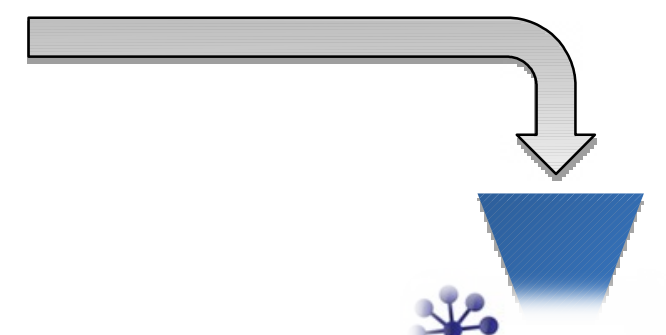

Ondex

Prot: PMN INIIMEDLINE:

PlantFoB GRAMENE Pfam

PO

phytozome GO 


\section{Connecting and querying}

Ondex - Yersion 0.3 (build null)

$-\mid \vec{a} \underline{x}$

Eile Edit View Appearance Iools Help

Enter search here...

Restrict by: Concept Class
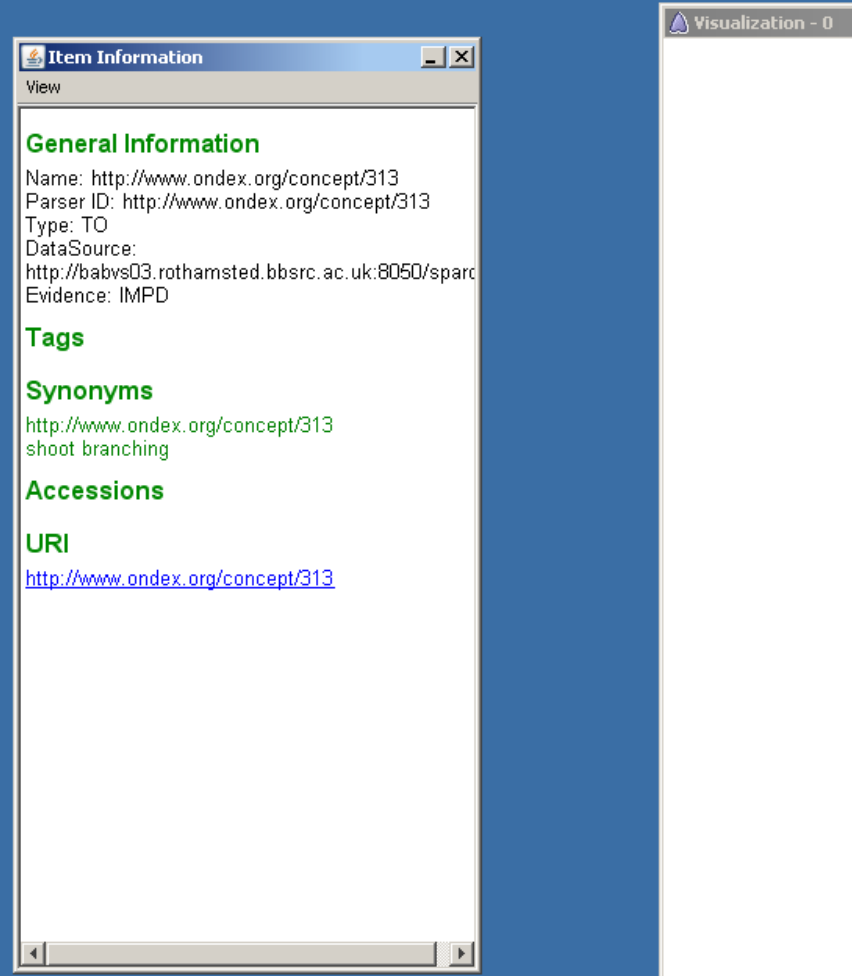

ONDEX $>$ construct $\{? \mathrm{x}$ ?y "shoot branching" $\}$

where \{graph 2g \{2x ?y "shoot branching" \})

ONDEX> 


\section{Right-click pre-defined queries}

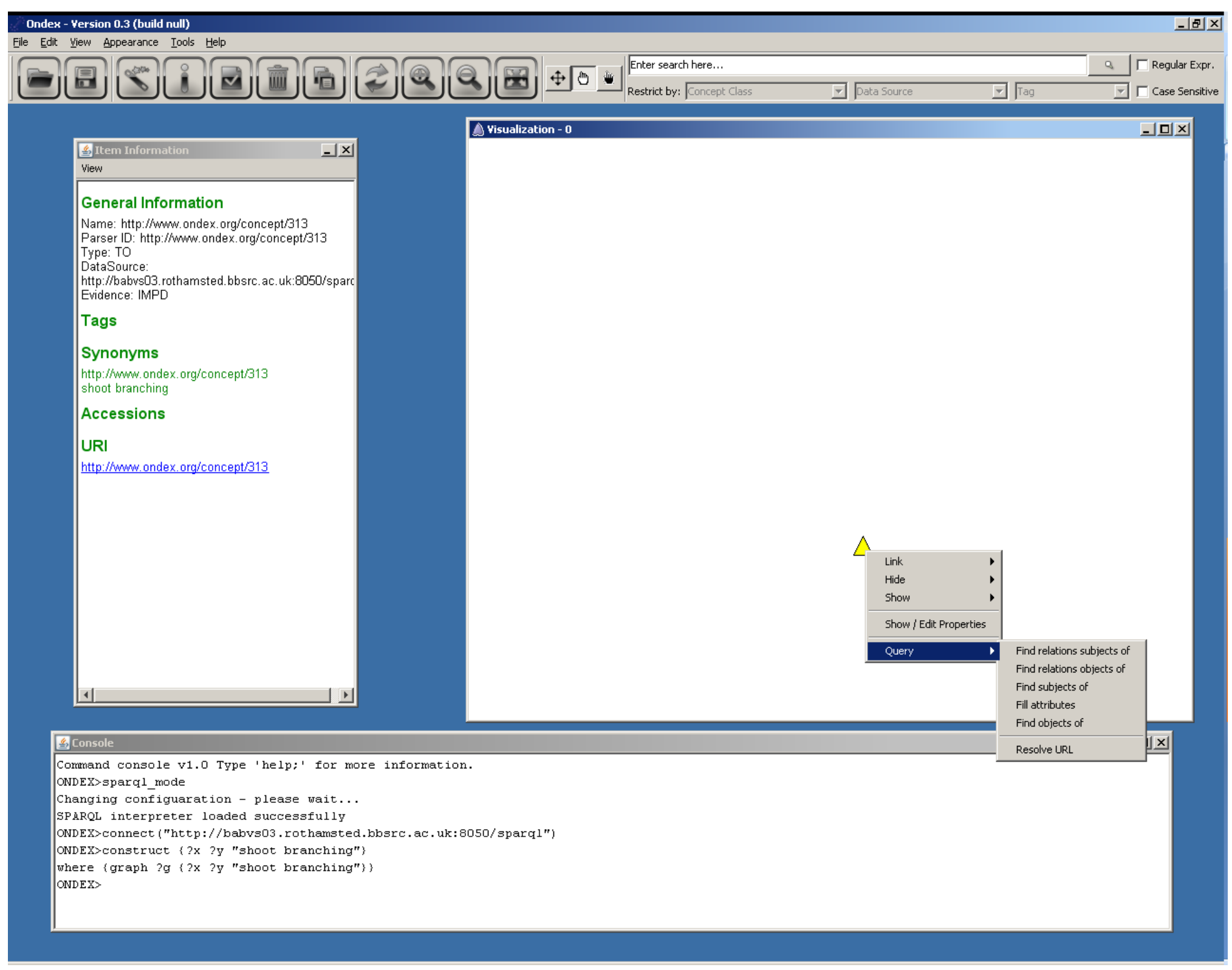




\section{Filling attributes}

Ondex - Yersion 0.3 (build null)

Eile Édit Yiew Appearance Iools Help

10

\section{重Ittem Information $-1 x$}

View

General Information

Name: http://mmww ondex. org/concept/292

Parser ID: 13130077

Type: Publication

DataSource: http://babvs03.rothamsted. bbsrc. ac.uk:8050/sparql Evidence: IMPD

\section{Annotation}

[NUCLEOTIDE SEQUENCE [MRNA], FUNCTION, TISSUE SPECIFICITY, DEVELOPMENTAL STAGE, MUTAGENESIS

OF ALA-49 AND ARG-50]

Tags

Synonyms

http://wwww. ondex. org/concept/292

http:/Wwiw. ondex
PMID:13130077

Accessions

http://babvs03.rothamsted.bbsrc.ac.uk:8050/sparql: 10.1073/PNAS. 193241410013130077

Publication

LAX and SPA: major regulators of shoot branching in rice. Keishi Komatsu; Masahiko Maekawa; Shin Ujiïe; Yuzuki Satake; Kuyo Furutan;; Hironobu Okamoto; Ko Shimamoto; Junko Kyozuk

\section{Year: 2003}

Published in: Proc. Natl. Acad. Sci. U.S.A.

The aerial architecture of plants is determined primarily by the initiation during embryog. Als is de by molecular gentic approaches using Arabidopsis little is known about the genetic mechanisms controlling axillary meristem initiation, mainly because of the insufficient number of mutants that specifically alter it. We identified the LAX PANICLE (LAX) and SMALL PANICLE (SPA) genes as the main regulators of axillary meristem formation in rice. LAX encodes a basic helix-loop-helix transcription factor and is expressed in the boundary between the shoot apical meristem and the region of new meristem formation. This pattern of $L A X$ expression was repeatedly observed in every axillary meristem, formation of the our formation of all types of axillary meristems throughout the
ontogeny of a rice plant. Ectopic LAX expression in rice caused pleiotropic effects, including dwarfing, an altered pattern of stem elongation, darker color, bending of the lamina joint, absence of the midribs of leaves, and severe sterility.

도․

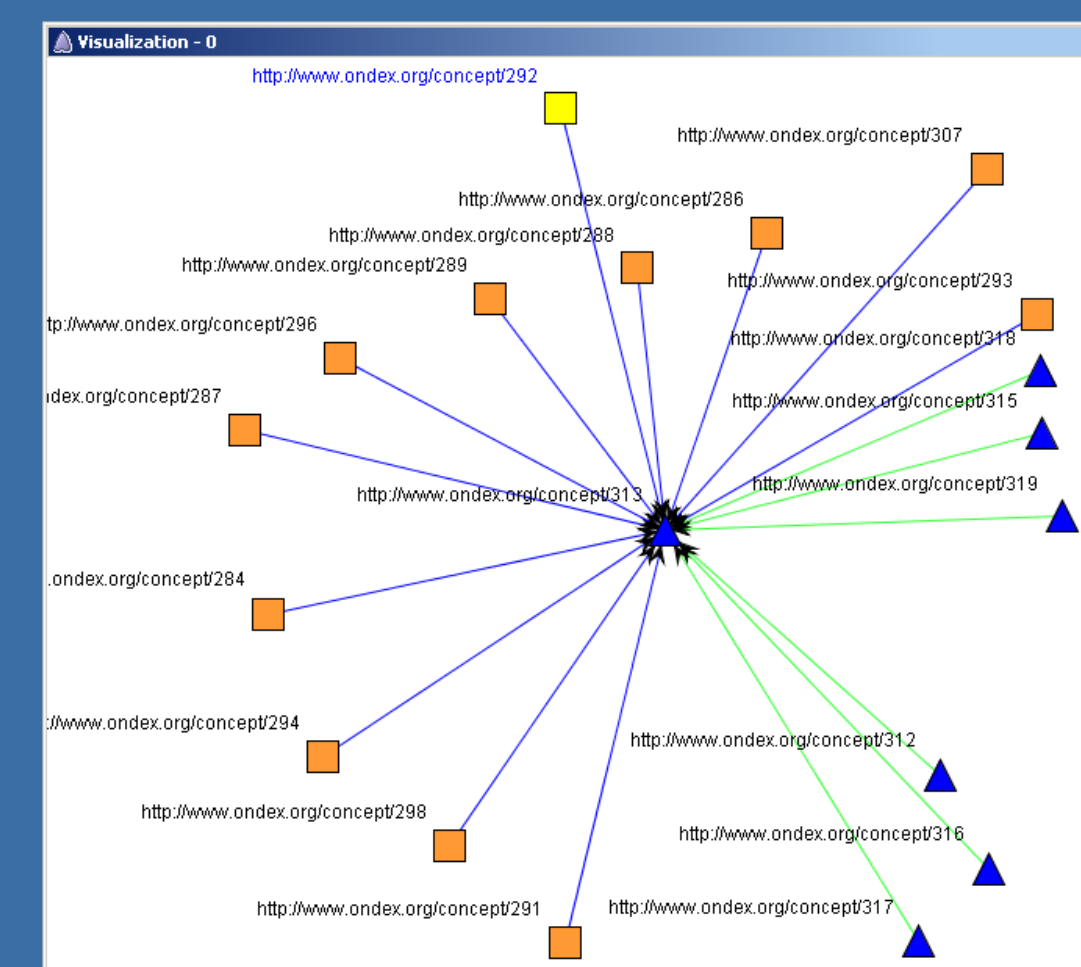




\section{Proteins related to shoot branching}

Ondex - Version 0.3 (build null)

Eile Edit View Appearance Iools Help एक

Enter search here.

\section{重Item Information}

General Information

Name: http://hwww. ondex. org/concept/159

Parser ID: LAX

DataSource: http://babvs03. rothamsted. bbsrc. ac. uk: 8050/sparq Evidence: IMPD

Tags

Synonyms

http://Wwhw ondex org/concept/159

AX1

LOC_Os01g61480

Os01g0831000

P0446G04.27

Transcription factor LAX PANICLE

\section{Accessions}

http://babvs03.rothamsted.bbsrc.ac.uk:8050/sparql: AP003252 (1)

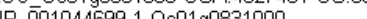

Taxonomy

39947

Aminoacid sequence (AA):

MHDPRGFP I HFQPYHL HPT AGGLGEGPNR

GGGRRRP GAK LSTDP QSUAA RERRRR ISDR

F RULRSLUP GGSHATTUSM LEQAIHYNKF

LKAQUTLHQA ALVQHEEGCQ HADUAAAFSA

MHRPGGAGDD DAGHTTLEHA

$\triangle$ Metadata Legen

$-\underline{-}$ 므 $|x|$ Concept classes | Data Sources | RelationTypes | EvidenceTypes |

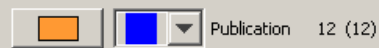

$\square \longdiv { \Delta \square }$ Tо $7(7)$

$\square \longdiv { P }$ Protein $8(8)$

Total Concepts 27 (27)

Copy to clipboard

\section{$\triangle$ Visualization - 0}
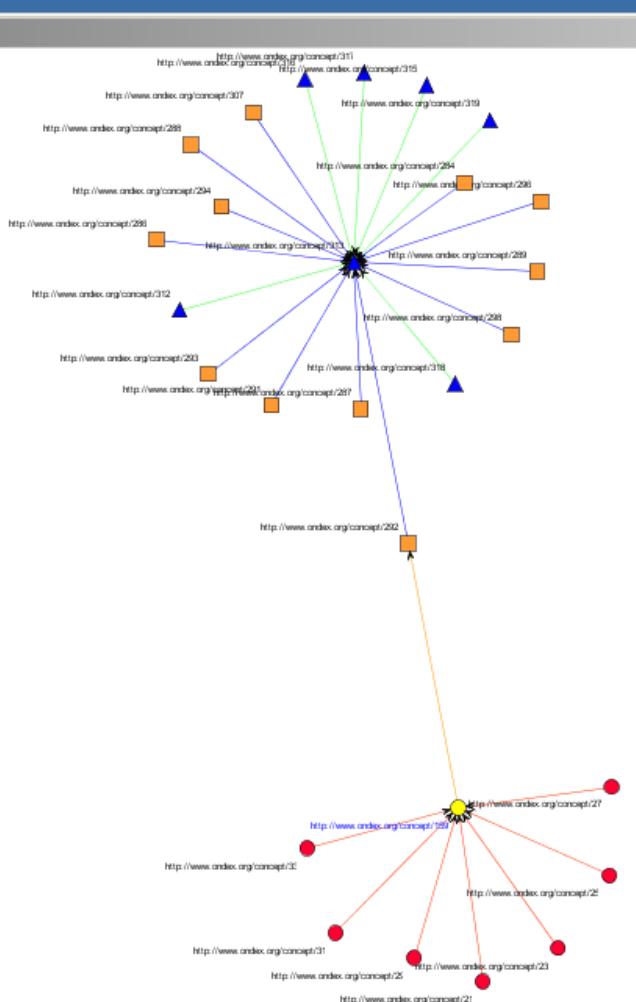

魚 Console

Cormand console v1.0 Type 'help;' for more information. ONDEX $>$ sparq1_mode

Changing configuaration - please wait..

SPARQL interpreter loaded successfully

ONDEX>connect ("http ://babvs03. rothamsted. bbsre . ac . uk : 8050/sparq1")

ONDEX $>$ construct $\{2 \mathrm{x}$ ?y "shoot branching" $\}$

where \{graph ?g \{? ? $\mathrm{y}$ "shoot branching"\}

ONDEX> 


\section{Colour proteins by taxonomy ID}

Ondex - Yersion 0.3 (build null)

Eile Edit Giew Appearance Iools Help

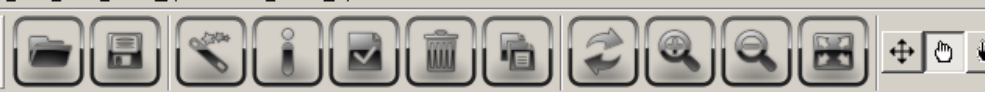

Fnter search here...

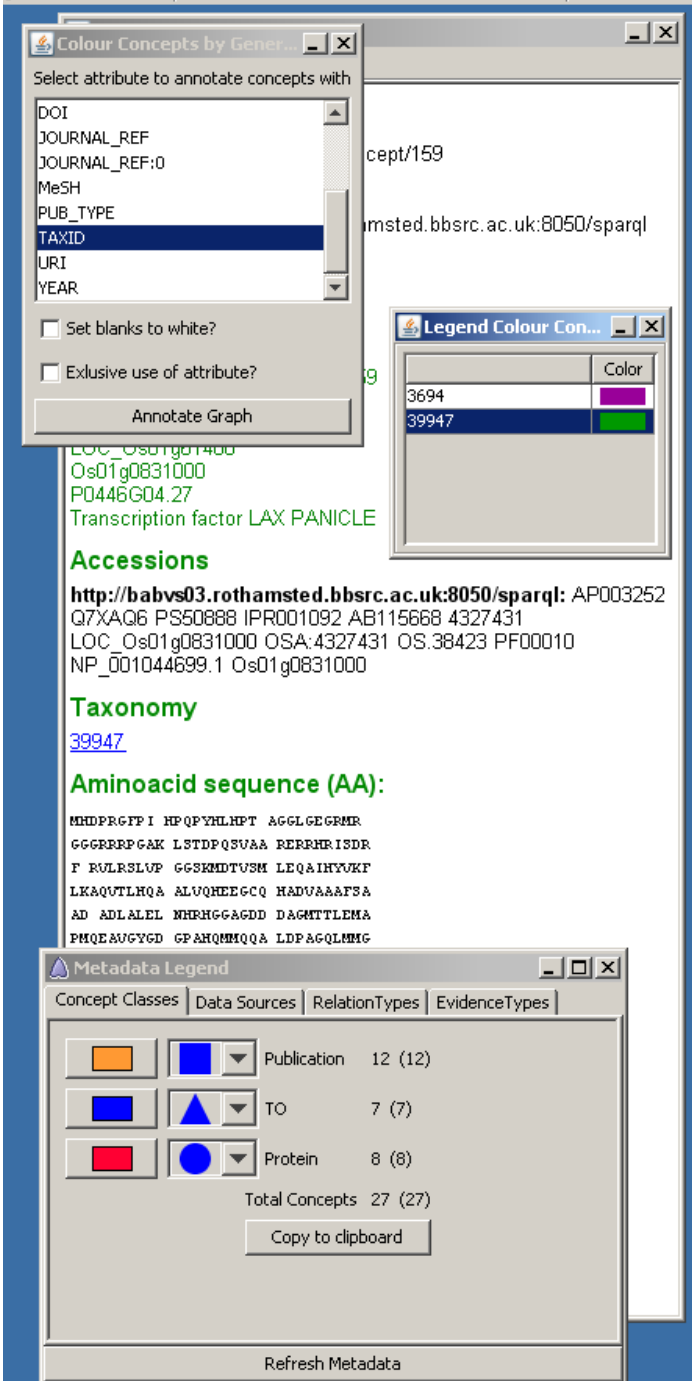

Aminoacid sequence (AA):

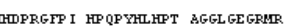

GGRRPP GAK LSTDP QSUAA RERRHR IBDE

RULRSLUP GGSKATTUSM LEQA THYTKF

$-|\square| x \mid$

Concept Classes | Data Sources | RelationTypes | EvidenceTypes |

- BBSRC

ONDEX $>$ construct $\{$ ? $2 \mathrm{y}$ "shoot branching" $\}$ $\triangle$ visualization - 0

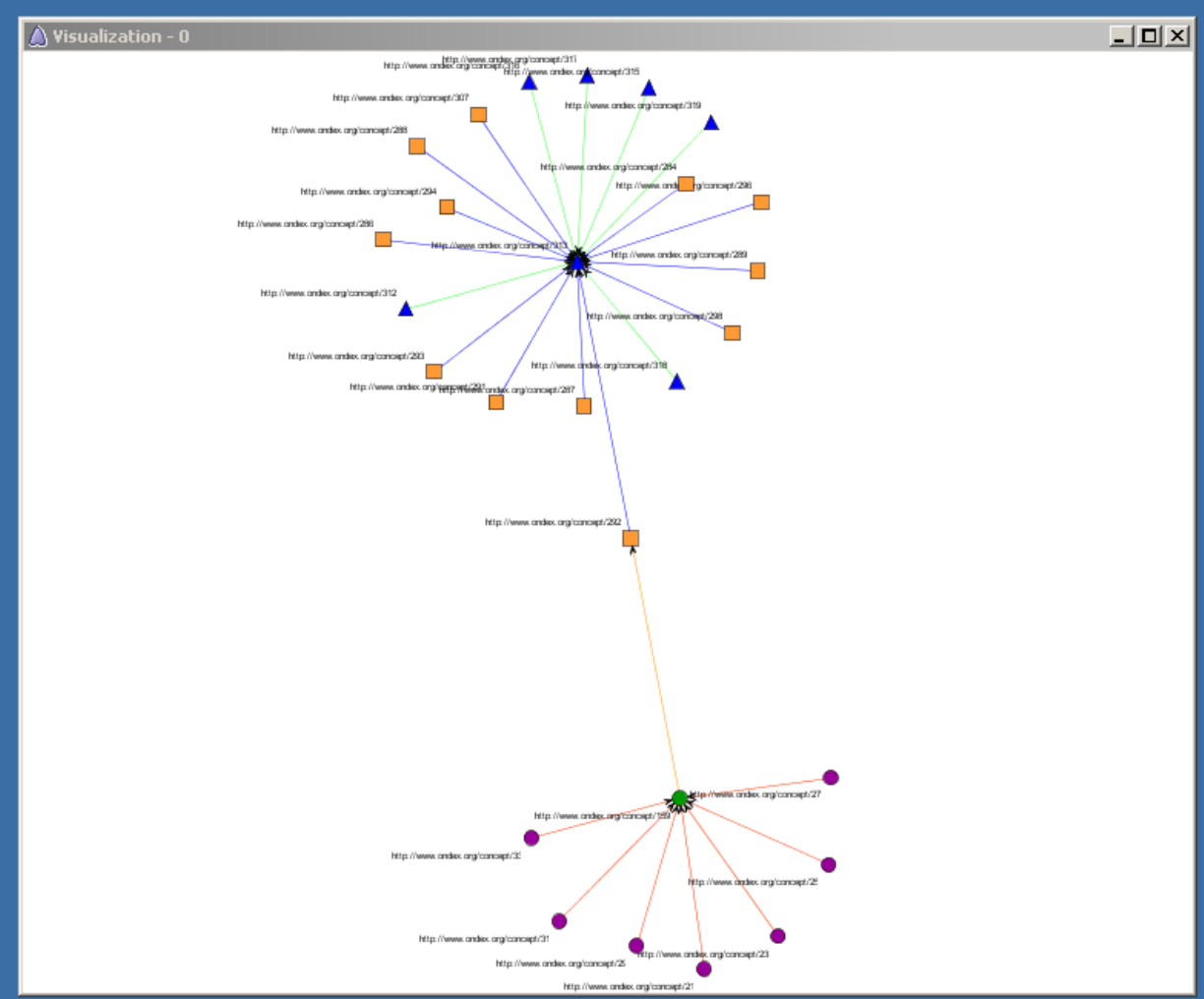

Cormand console v1.0 Type ' help:' for more information. ONDEX $>$ sparq1_mode

ONDEX>sparq1_mode

hanging conflguaration - please wait...

PARQL interpreter loaded successfully

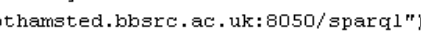

where \{graph ?g \{? $2 \mathrm{y}$ "shoot branching"\}

ONDEX> 


\section{Scale relation based on BLAST}

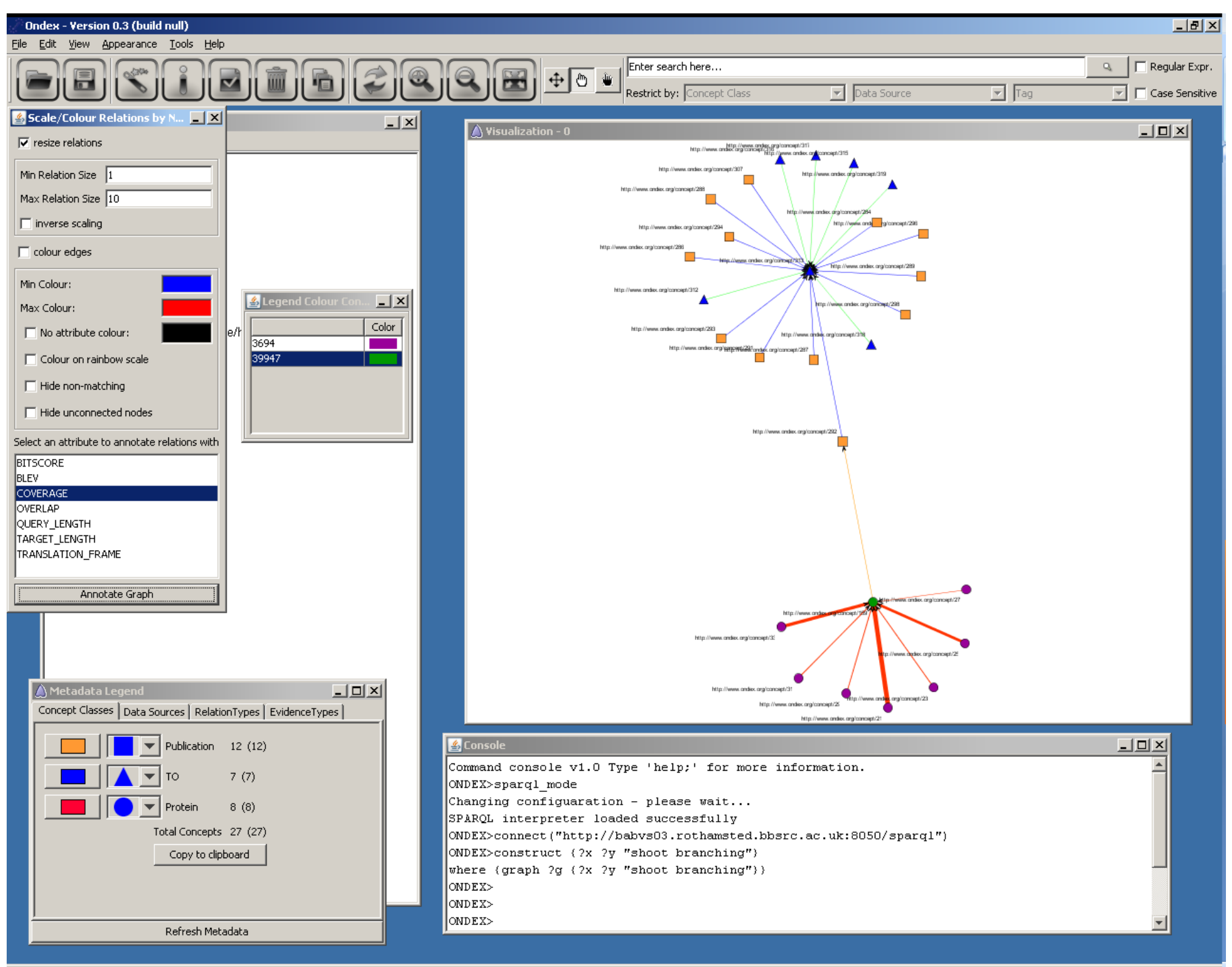




\section{Acknowledgements}

$\circ$ Artem Lysenko

- Andrea Splendiani

o Matthew Pocock

○ Chris Rawlings 